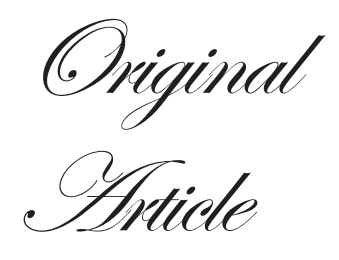

\title{
Indocyanine green clearance test (using spectrophotometry) and its correlation with Model for End Stage Liver Disease (MELD) score in Indian patients with cirrhosis of liver
}

\author{
Sachin Gupta, ${ }^{1}$ Yogesh Chawla, ${ }^{1}$ Jyotdeep Kaur, ${ }^{2}$ Roli Saxena, ${ }^{2}$ Ajay \\ Duseja, ${ }^{1}$ RK Dhiman, ${ }^{1}$ Narendra S Choudhary ${ }^{1}$
}

\begin{abstract}
Departments of Hepatology ${ }^{1}$ and Biochemistry, ${ }^{2}$

Post Graduate Institute of Medical

Correspondence:

Dr. Yogesh Chawla

Email: ykchawla@gmail.com
\end{abstract}

ABSTRACT

Background: Child Turcotte Pugh (CTP) score and Model for End Stage Liver Disease (MELD) are used commonly to assess the prognosis of liver disease but the disadvantage of these static tests is their inability to identify the functional reserve of the liver. Among all quantitative liver function tests indocyanine green (ICG) clearance test is most widely used and has been used to determine operative risk before hepatectomy and to assess prognosis of patients with cirrhosis.

Aim: To correlate indocyanine green (ICG) clearance test with MELD score in patients with cirrhosis of liver.

Methods: Forty patients with cirrhosis of liver were included and divided into two groups according to their CTP scores. Group A had 20 patients with CTP class A and group B had 20 patients with CTP class B. After ICG injection, ICG retention at 15 minutes (ICGR15) and ICG clearance rate were calculated.

Results: In group A, the mean ICGR15 was $32.86 \% \pm 6.4 \%$ while in group B it was $51.08 \% \pm$ $12.8 \%$ ( $\mathrm{p}<0.001$ ). ICG clearance rates were $4.3 \% \pm 2.8 \%$ and $3.5 \% \pm 3.8 \%$ per minute in group A and B respectively. MELD score had a strong positive correlation with ICGR15 but a negative correlation with ICG clearance rate. On ROC curve analysis, AUC for MELD was 0.805 vs. 0.88 for ICGR15 in assessing prognosis of patients with cirrhosis. The sensitivity and specificity of MELD score was $60 \%$ and $80 \%$ respectively while that of ICGR15 was $85 \%$ and $90 \%$ respectively.

Conclusion: ICGR15 has a higher sensitivity and specificity than MELD score in assessing the prognosis of patients with cirrhosis of liver.

KEYWORDS: Cirrhosis of liver, Child Turcott Pugh score, Model for End Stage Liver Disease, indocyanine green clearance test.

\section{Introduction}

Liver function tests can be broadly divided into static tests and dynamic tests i.e. quantitative liver function tests. Child Turcotte Pugh (CTP) and Model for End-stage Liver Disease
(MELD) scores are commonly used to assess the prognosis of liver disease but major disadvantage of static liver function tests is their inability to identify the functional reserve of the 
liver. ${ }^{1}$ The MELD score has emerged as a useful tool for estimating the mortality in patients awaiting liver transplantation. It was implemented in 2002 by the United Network for Organ Sharing (UNOS) as the criterion for organ allocation in patients with chronic liver disease awaiting liver transplantation.

Dynamic or quantitative liver function tests are improvement of static tests but are generally cumbersome and not widely available. Dynamic tests measure the clearance of tracer substances and provide considerably more information than static tests. Indocyanine green (ICG) is a synthetic dye that is eliminated by the liver without extrahepatic metabolism and excretion, and its blood clearance has been applied to determine the operative risk before hepatectomy, ${ }^{2}$ as well as to evaluate donor liver function in transplantation. ${ }^{3,4}$ ICG clearance test has also been found to be useful for assessment of prognosis of patients of cirrhosis but there is no data of indocyanine green clearance test from India. ${ }^{5}$

Thus, aim of this study was to correlate indocyanine green clearance test with Child Turcotte Pugh (CTP) score and Model for End Stage Liver Disease (MELD) score in patients with liver cirrhosis.

\section{Methods}

Patients

This was a prospective cohort study. Study population comprised of cirrhotic patients admitted to the Department of Hepatology or those attending the Liver Clinic of the Post Graduate Institute of Medical Education and Research, from July 2009 to December 2010. The diagnosis of cirrhosis was based on clinical, biochemical, radiological and endoscopic features. A total of 82 patients were evaluated and 40 of them were included in the study. Out of 40 cirrhotics, 20 patients were in CTP class A and 20 patients were in CTP class B. Exclusion criteria included: Child's $\mathrm{C}$ cirrhosis, active upper gastrointestinal bleed, hepatic encephalopathy over last 2 weeks, hepatocellular carcinoma, pregnant females and patients with history of allergy to dyes or iodides (ICG injection contains sodium iodide). Forty two patients were excluded, 31 were in CTP C class, 11 had encephalopathy, 7 had HCC and 4 had active upper GI bleed ( 7 patients who had encephalopathy and 4 who had HCC were in CTP C class). The study protocol was approved by ethical committee of the institute and informed consent was taken from all the patients.

\section{Clinical and laboratory assessment}

Clinical examination included a thorough general physical and systemic examination. Laboratory investigations like complete hemogram, serum electrolytes, renal and liver function tests, and complete coagulogram were done in all the cases. Upper gastrointestinal endoscopy was performed to look for the presence of esophageal varices. Severity of disease was determined by Child Turcotte Pugh's classification and MELD score.

\section{ICG clearance test}

A solution of commercially available indocyanine green (Aurogreen from Aurolab, Madurai, India) was prepared by diluting the dye into distilled water to a final concentration of $5 \mathrm{mg} / \mathrm{ml}$. A bolus injection of $0.5 \mathrm{mg} / \mathrm{kg}$ of ICG solution was injected to the patients through a peripherally placed venous cannula. After injection, venous blood samples were collected at 5 minute intervals for 15 minutes. The concentration was measured photometrically at $805 \mathrm{~nm}$ using a spectrophotometer (Beckmann Coulter DU 640). The dye clearance was measured as a product of volume of distribution and elimination rate (VD $\times \mathrm{K})$. ICG concentration in the plasma was calculated at every 5 minutes interval for 15 minutes after injection to calculate the clearance and retention of ICG at 15 minutes i.e. ICGR15.

\section{Statistical analysis}

The statistical analysis was carried out using Statistical Package for Social Sciences (SPSS Inc., Chicago, IL, version 13.0). All quantitative variables were estimated using mean, median and standard deviation. For normally distributed data, means were compared using student t-test for two groups. For skewed data, Mann Whitney test was applied for two groups. Qualitative or categorical variables were described as frequencies and proportions. Proportions were compared using Chi square or Fisher's exact test. ROC Curves were used to look for the cut off points for different variables.

\section{Results \\ Patient characteristics}

A total of 40 patients of liver cirrhosis who satisfied all the eligibility criteria, were included in the study. Patients were 
divided into two groups based on their CTP scores. Group A had 20 patients with CTP class A and group B had 20 patients with CTP class B. Demographic characteristics of patients are shown in Table 1.

The various etiologies of cirrhosis in our study included alcohol, HBV, HCV and cryptogenic (non-B non-C and nonalcoholic groups in whom other etiologies like autoimmune disorders and Wilson's disease were adequately ruled out). In group A, 7 patients (35\%) had cryptogenic cirrhosis, 6 patients (30\%) each had HBV and HCV related cirrhosis and only 1 patient $(5 \%)$ had alcohol related cirrhosis. In group B, the most common etiology of cirrhosis was alcohol (50\%), four patients $(20 \%)$ had cryptogenic cirrhosis and 3 patients (15\%) each had HBV and HCV related cirrhosis, respectively. The difference in etiology in both the groups was significant for alcoholic cirrhosis only $(p<0.001)$ but not for the other etiologies. In patients of group A, the mean MELD score was $9.7 \pm 2.32$ and in group B it was $13.3 \pm 3.8(\mathrm{p}=0.001)$ (Table 2).

Table 1: Baseline characteristics of 40 patients with cirrhosis of liver

\begin{tabular}{lccc}
\hline Parameter & Group- A & Group B & p value \\
\hline $\begin{array}{l}\text { Number of patients } \\
\text { Age in years (Mean) }\end{array}$ & 20 & 20 & \\
Gender & 47.35 & 48 & 0.817 \\
- Males (\%) & $16(80 \%)$ & $19(95 \%)$ & 0.151 \\
- Females (\%) & $4(20 \%)$ & $1(5 \%)$ & \\
Etiology of cirrhosis & & & \\
- Alcohol & $1(5 \%)$ & $10(50 \%)$ & $<0.001$ \\
- HBV & $6(30 \%)$ & $3(15 \%)$ & 0.45 \\
- HCV & $6(30 \%)$ & $3(15 \%)$ & 0.45 \\
- Cryptogenic & $7(35 \%)$ & $4(20 \%)$ & 0.48 \\
Ascites & & & \\
- Present (\%) & 0 & $3(15 \%)$ & 0.231 \\
- Absent (\%) & $20(100 \%)$ & $17(85 \%)$ & \\
History of GI bleed & $2(10 \%)$ & $4(20 \%)$ & 0.66 \\
Varices & & & \\
- Absent & $3(15 \%)$ & $3(15 \%)$ & 0.721 \\
- Large & $5(25 \%)$ & $3(15 \%)$ & \\
- Small & $12(60 \%)$ & $14(70 \%)$ & \\
\hline
\end{tabular}

Table 2: CTP score, ICGR15, ICG clearance and MELD score in 40 patients of cirrhosis

\begin{tabular}{lccc}
\hline Parameter & Group A & Group B & p value \\
\hline CTP score & 5.4 & 7.85 & $<0.001$ \\
ICGR15 & & & \\
- Mean & $32.86 \%$ & $51.08 \%$ & $<0.001$ \\
- Range & $(23.6 \%-46.04 \%)$ & $(26 \%-68.9 \%)$ & \\
ICG Clearance & & & \\
- Mean & $4.36 \%$ & $3.54 \%$ & 0.04 \\
- Range & $(1.6 \%-13 \%)$ & $(0.09 \%-18.2 \%)$ & \\
MELD & 9.7 & 13.3 & 0.001 \\
\hline
\end{tabular}

\section{ICGR 15}

In group A, the range of ICGR15 was found to be between $23.6 \%$ and $46 \%$, with a mean value of $32.86 \pm 6.4 \%$. In group B, the range of ICGR 15 was between $26 \%$ and $69 \%$, with a mean of $51.08 \pm 12.8 \%(\mathrm{p}<0.001)$.

\section{ICG clearance}

In group A, ICG clearance rate was in the range of 1.6 to $13 \%$ per min with a mean of $4.3 \pm 2.8 \%$ per min. In group $B$, the range was 1.9 to $18.2 \%$ per min with a mean of $3.5 \pm 3.8 \%$ per min $(\mathrm{p}=0.04)$.

\section{Correlation between CTP score and ICGR15}

There was a strong positive correlation between CTP score and ICGR 15 i.e. as the CTP score increased, the value of ICGR15 also increased. In other words, as the liver functions worsened, there is more retention of ICG (Figure 1). The correlation coefficient between CTP score and ICGR15 was 0.658 ( $\mathrm{p}<0.001)$.

\section{Correlation between MELD score and ICGR15}

The MELD score and ICGR15 demonstrated a strong positive correlation i.e. as the MELD score increased, the value of ICGR15 increased. In other words, as the liver function worsens, there is more retention of ICG (Figure 2). The correlation coefficient between MELD score and ICGR15 was $0.482(\mathrm{p}=$ 0.002).

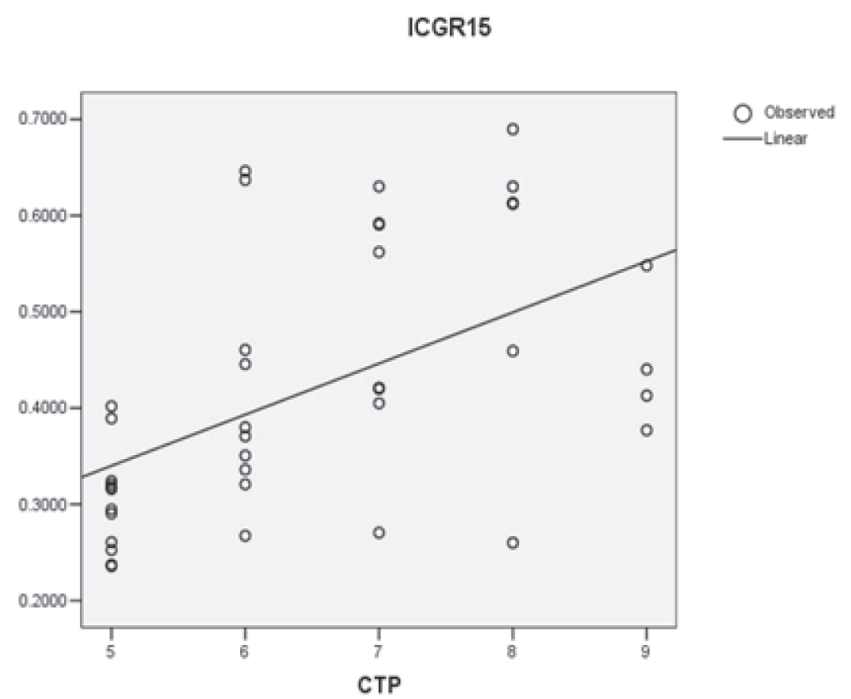

Figure 1: Correlation between CTP score and ICGR15 
Comparison of MELD score with ICGR15 in assessing prognosis of patients of cirrhosis

To assess the prognosis of patients with cirrhosis, ROC curve analysis was done. The area under curve (AUC) for MELD and ICGR15 were 0.805 and 0.88 respectively (Figure 3). The sensitivity and specificity of MELD score was $60 \%$ and $80 \%$, respectively, and that for ICGR 15 was $85 \%$ and $90 \%$, respectively. Thus we found that in comparison to the MELD score, ICGR 15 had greater sensitivity and specificity in assessing the prognosis (in terms of estimated survival based on CTP class) of patients with cirrhosis.

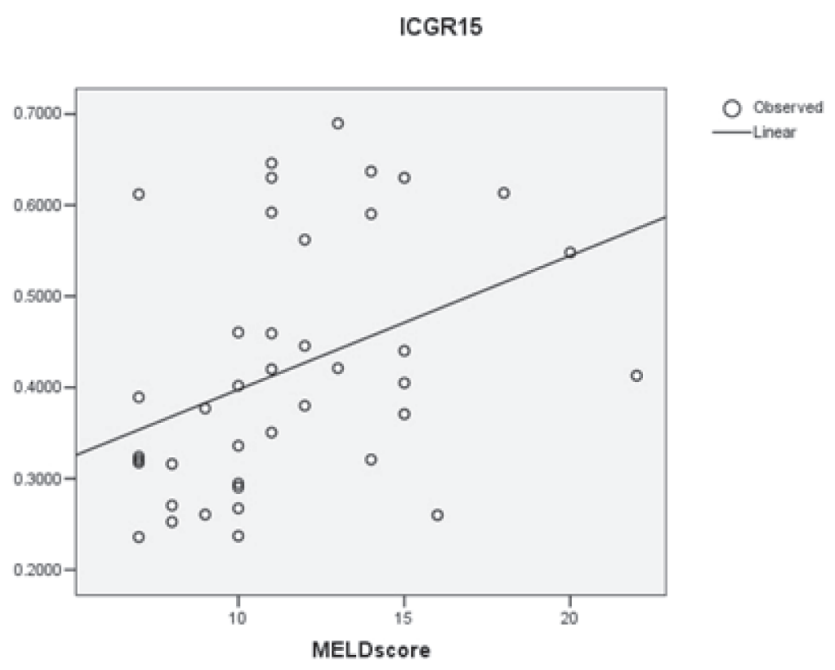

Figure 2: Correlation between MELD score and ICGR15

ROC Curve

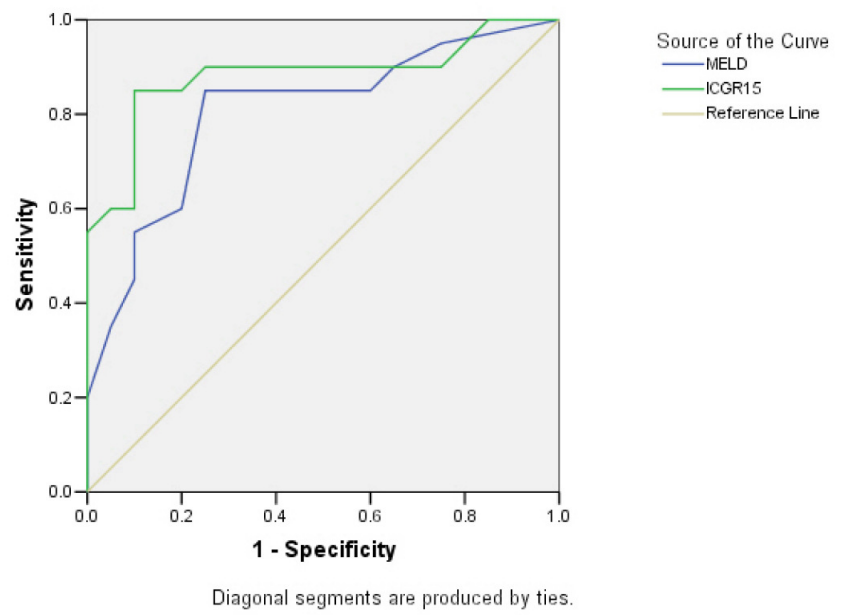

Figure 3: Receiver Operative Characteristic (ROC) Curve for MELD score and ICGR15

\section{Discussion}

To assess the prognosis of cirrhosis, the CTP score has been used traditionally. The MELD score was formulated to predict three month mortality for cirrhotic patients undergoing transjugular intrahepatic portosystemic shunt. ${ }^{6}$ The MELD scoring system is now being used widely in patients with end stage cirrhosis awaiting liver transplantation. ${ }^{7}$ MELD has been validated on a large series of patients with liver diseases of various etiologies and severity. ${ }^{8}$ Although MELD score is a good prognostic scoring system and considered better than the CTP score for patients with cirrhosis, it has several limitations. First, it does not include ascites and encephalopathy which itself are signs of poor prognosis in these patients. Second, value of prothrombin time and INR varies from laboratory to laboratory depending upon the reagent and method used. Third, serum creatinine value is sometimes altered with the use of diuretics. Hence there arises a need to modify MELD or to develop a better score. Addition of $\mathrm{Na}^{+}$into the MELD (MELD-Na) has been proposed but not yet validated. MELD-Na has been successfully evaluated as a better score in patients with ascites. It adds up to 13 points to the MELD score, but only for sodium levels below normal, a typical feature of decompensated cirrhosis found in $30 \%$ of the pre-transplant population. ${ }^{9}$

CTP and MELD are not useful to assess the hepatic functional reserve. Dynamic liver function tests are better markers of functional capacity of the liver and thus have advantage over routine liver function tests. There are many quantitative liver function tests which have been used for this purpose but the most commonly studied and most widely used is the ICG clearance test. It is used more extensively in eastern countries e.g. Taiwan and China than in the West, probably because of a higher incidence of hepatocellular carcinoma and is being used mostly to assess pre-resection hepatic function.

Studies have indicated that the ICG clearance test is a sensitive indicator for evaluating liver function. ICG clearance test has also been a good predictor of survival in cirrhotic patients and is used routinely at some centers before liver surgery. There are many studies published in literature on ICG clearance in various situations e.g. to assess liver function before resection, to asses liver function in critically ill patients, ${ }^{10}$ in acute liver failure ${ }^{11}$ and to assess graft and donor function in liver transplantation, ${ }^{12}$ but there are only few studies of indocyanine green clearance test for assessing the prognosis of patients with cirrhosis and its correlation with the CTP and MELD scores. ${ }^{8,13,14}$ There is no study published in the literature on ICG clearance test in cirrhotics from India.

Patients included in our study were cirrhotics of CTP class $\mathrm{A}$ and $\mathrm{B}$ and not that of CTP C, therefore ascites was present 
only in 3 out of 40 patients $(7.5 \%)$. None of the patients had clinical jaundice or overt encephalopathy or active gastrointestinal bleeding. We did not include patients of CTP C score because we did not have any data about safety of indocyanine green dye in these patients.

In our study, the mean of ICGR15 in CTP A group was 32\% while in CTP B group it was $51 \%$. These results are similar to studies by Gilbert et $\mathrm{al}^{14}$ in 1959 and Shimada et $\mathrm{al}^{14}$ in 1965. The method of estimation of indocyanine green clearance used in these studies was conventional spectrophotometry, the same as employed in our study. In contrast, recent studies on indocyanine green clearance test in patients of cirrhosis have used pulse dye densitometry and have different values of indocyanine green clearance and indocyanine retention at 15 minutes. In a study by Sheng et $\mathrm{al}^{5}$ the mean ICGR 15 in patients with CTPA was $7.3 \%$ and in CTP B it was $13.7 \%$ (much lower than our study). The mean MELD score in our study of patients with CTP A and B was 9.7 and 13.3 respectively while in the Sheng study, the MELD scores were 3.8 and 10.8, respectively. This suggests that the patients in our study had a higher MELD score than the patients in above mentioned study and that may be one reason why there was a difference in values of ICGR 15 in both the studies. It is also possible that the different cut-off values in our patients could be because of differences among different races with some contribution of anthropometric variability. There may be a different cut-off in the Indian population but to prove this, further studies are needed.

There was a strong positive correlation between the CTP and MELD scores with ICGR15 and a negative correlation between the CTP and MELD scores with ICG clearance in our study. The correlation was stronger for ICGR15 than ICG clearance rate, suggesting that the ICGR 15 is a more convenient and appropriate indicator to evaluate liver function in patients with liver cirrhosis. This finding is similar to those of Sheng et al who found that in comparison to ICG clearance, ICGR15 was a better indicator for assessment of liver function.

On receiver operative characteristic (ROC) analysis, the area under curve (AUC) for ICGR15 was better than the AUC of MELD score (AUC: 0.88 vs. 0.80). The sensitivity and specificity of MELD score was $60 \%$ and $80 \%$, respectively, but for ICGR15 it was $85 \%$ and $90 \%$, respectively. Thus in comparison to the MELD score, ICGR15 had greater sensitivity and specificity in assessing the prognosis of patients with cirrhosis. In contrast Stauber et $\mathrm{al}^{13}$ found a superior diagnostic accuracy for MELD as compared with ICG clearance in predicting 90 day survival in patients of cirrhosis. In a recent study by Alexander et $\mathrm{al}^{16}$ incorporation of ICG into the MELD score adds an estimation of liver blood flow and renders the new score 'MELD-ICG' more accurate in predicting survival than the MELD and MELD-Na.

Our study has few limitations like small sample size, exclusion of CTP C cirrhotics and the use of conventional spectrophotometry rather than pulse dye densitometry. In conclusion, this study shows that ICGR15 has a good prognostic value in patients of cirrhosis and has higher sensitivity and specificity than the CTP score and MELD score in assessing the prognosis of patients with cirrhosis.

\section{References}

1. Jalan R, Hayes PC. Review article: quantitative tests of liver function. Aliment Pharmacol Ther. 1995:9:263-70.

2. Mullin EJ, Metcalfe MS, Maddern GJ. How much liver resection is too much? Am J Surg. 2005;190:87-97.

3. Kamei H, Fujimoto Y, Nagai S, Suda R, Yamamoto H, Kiuchi T. Impact of non-congestive graft size in living donor liver transplantation: new indicator for additional vein reconstruction in right liver graft. Liver Transpl. 2007;13:1295-301.

4. Hsieh CB, Chen CJ, Chen TW, Yu JC, Shen KL, Chang TM, et al. Accuracy of indocyanine green pulse spectrophotometry clearance test for liver function prediction in transplanted patients. World J Gastroenterol. 2004;10:2394-6.

5. Sheng QS, Lang R, He Q, Yang YJ, Zhao DF, Chen DZ. Indocyanine green clearance test and model for end-stage liver disease score of patients with liver cirrhosis. Hepatobiliary Pancreat Dis Int. 2009;8:46-9.

6. Kamath PS, Wiesner RH, Malinchoc M, Kremers W, Therneau TM, Kosberg CL, et al. A model to predict survival in patients with end-stage liver disease. Hepatology. 2001;33:464-70.

7. Christensen E. Prognostic models including the Child-Pugh, MELD and Mayo risk scores - where are we and where should we go? J Hepatol. 2004;41:344-50.

8. Durand F, Valla D. Assessment of the prognosis of cirrhosis: Child-Pugh versus MELD. Hepatology. 2005;42:S100-7.

9. Kim WR, Biggins SW, Kremers WK, Wiesner RH, Kamath PS, Benson JT, et al. Hyponatremia and mortality among patients on the liver-transplant waiting list. $N$ Engl J Med. 2008;359:1018-26.

10. Sakka SG, Reinhart K, Meier-Hellmann A. Prognostic value of the indocyanine green plasma disappearance rate in critically ill patients. Chest. 2002;122:1715-20.

11. Ramsøe K, Andreasen PB, Ranek L. Functioning liver mass in uncomplicated and fulminant acute hepatitis. Scand $J$ Gastroenterol. 1980;15:65-72.

12. Kakizoe S, Yanaga K, Starzl T E, Demetris AJ. Evaluation of protocol before transplantation and after reperfusion biopsies from human orthotopic allografts: considerations for preservation and early immunological injury. Hepatology. 1990;11:932-41.

13. Stauber RE, Wagner D, Stadlbauer V, Palma S, Gurakuqi G, Kniepeiss D, et al. Evaluation of indocyanine green clearance 
and model for end stage liver disease for estimation of short-term prognosis in decompensated cirrhosis. Liver Int. 2009;29:1516-20.

14. Zipprich A, Kuss O, Rogowski S, Kleber G, Lotterer E, Seufferlein $\mathrm{T}$, et al. Incorporating indocyanin green clearance into the Model for End Stage Liver Disease (MELD-ICG) improves prognostic accuracy in intermediate to advanced cirrhosis.
Gut. 2010;59:963-8.

15. Cherrick GR, Stein SW, Leevy CM, Davidson CS. Indocyanine green: observations on its hysical properties, plasma decay and hepatic extraction. J Clin Invest. 1960;39:592-600.

16. Shimada Y, Maeda T, Kizu H et al. A study on indocyanine green clearance test in liver disease. J Gastroenterol. 1965;1:58. 